\title{
Correlation times in stochastic equations with delayed feedback and multiplicative noise
}

\author{
Mathieu Gaudreault, ${ }^{1}$ Juliana Militão Berbert, ${ }^{1,2}$ and Jorge Viñals ${ }^{1}$ \\ ${ }^{1}$ Department of Physics, McGill University, Montreal, Quebec, Canada H3A 2 T8 \\ ${ }^{2}$ Instituto de Física Teórica (IFT), Universidade Estadual Paulista (UNESP), Caixa Postal 70532-2, 01156-970 São Paulo, São Paulo, Brazil
}

(Received 30 June 2010; published 11 January 2011)

\begin{abstract}
We obtain the characteristic correlation time associated with a model stochastic differential equation that includes the normal form of a pitchfork bifurcation and delayed feedback. In particular, the validity of the common assumption of statistical independence between the state at time $t$ and that at $t-\tau$, where $\tau$ is the delay time, is examined. We find that the correlation time diverges at the model's bifurcation line, thus signaling a sharp bifurcation threshold, and the failure of statistical independence near threshold. We determine the correlation time both by numerical integration of the governing equation, and analytically in the limit of small $\tau$. The correlation time $T$ diverges as $T \sim a^{-1}$, where $a$ is the control parameter so that $a=0$ is the bifurcation threshold. The small- $\tau$ expansion correctly predicts the location of the bifurcation threshold, but there are systematic deviations in the magnitude of the correlation time.
\end{abstract}

DOI: 10.1103/PhysRevE.83.011903

PACS number(s): 87.16.Yc, 02.30.Ks, 05.10.Gg, 05.70.Ln

\section{INTRODUCTION}

The correlation time of a nonlinear stochastic differential equation that includes delayed feedback and multiplicative noise is studied near its bifurcation line away from the trivial state. We focus on a nonlinear model of a stochastic differential equation that models a feedback loop motif of gene regulation network. We show that the correlation time diverges at threshold, consistent with a sharp bifurcation. An approximate expression for the correlation time valid for small time delay is provided that correctly predicts the location of the bifurcation threshold. However, the value of the analytical prediction of the correlation time does not agree with our numerical determination for finite $\tau$.

Analytic results describing stochastic differential delay equations are rare due to their non-Markovian nature. The assumption of independence between the state variable at time $t$ and its value at time $t-\tau$, where $\tau$ is the delay, is thus an often used approximation. For example, this decomposition is used to obtain an analytic solution to a master equation modeling protein degradation in [1]. This assumption of independence is valid in a region of parameter space in which the correlation time of the dynamical variables is smaller than other time scales in the system arising from the delay. Indeed, for sufficiently large values of the delay, one can safely assume that the state variable has lost memory of its past value and any delay term might act simply as another source of noise. We note, however, that close to a bifurcation the correlation time may diverge. The resulting non-Markovian nature of the model must be taken into account for any analysis thereafter as the state at time $t$ is correlated with the state at time $t-\tau$.

We focus in this paper on the normal form for a pitchfork bifurcation augmented with multiplicative or parametric noise and delay as described in [2]. The dynamical variable $x(t)$ obeys

$$
\dot{x}(t)=a x(t)+b x(t-\tau)-x^{3}(t)+x(t) \xi(t),
$$

where the constant $a$ plays the role of a control parameter, $b$ is the intensity of a feedback loop of delay $\tau>0$, and $\xi(t)$ is a white, Gaussian noise with mean $\langle\xi(t)\rangle=0$ and correlation $\left\langle\xi(t) \xi\left(t^{\prime}\right)\right\rangle=2 D \delta\left(t-t^{\prime}\right)$. The initial condition is a function $\phi(t)$ specified on $t=[-\tau, 0]$. Equation (1) is understood under the Stratonovich interpretation of stochastic calculus [3]. This interpretation is appropriate when white noise can be considered as the limiting case of the colored noise actually existing in the system [4]. This observation has been confirmed experimentally [5].

The bifurcation diagram of Eq. (1) has been determined numerically in [2]. Two bifurcation lines were obtained: a pitchfork bifurcation and a Hopf bifurcation. It was shown that the location of the bifurcation threshold depends on the intensity of the parametric noise. Analytical approximations for the bifurcation lines have been given in [6] under the assumption that the delay time is small compared to other characteristic time scales of the system. The pitchfork bifurcation line separates exponentially decaying and growing solutions when $b \tau>-1$. On the other hand, delayed feedback induces oscillations and the bifurcation is of the Hopf type when $b \tau<-1$. The two bifurcation lines intersect at $(a, b)=$ $(1 / \tau-D,-1 / \tau)$. We found that for small time delay the solution $x=0$ is stable when

$$
a<-b[1+\tau(b+D)](1+b \tau)^{-1}
$$

for the pitchfork branch $(b \tau>-1)$, and

$$
\tau \sqrt{b^{2}-(a+D)^{2}}<\cos ^{-1}[-(a+D) / b]
$$

for the Hopf branch $(b \tau<-1)$. Exponential growth saturates due to nonlinearities.

The bifurcation diagram of Eq. (1) without delay $(b=0)$ is known [7-9]. The stochastic threshold is located at $a_{c}=0$, at which point the stationary distribution function changes from a delta function at the origin to a power law with an exponential cutoff at large $x$. The correlation function of the linear counterpart of Eq. (1) but with additive noise is known [10,11]. The correlation function and correlation time of Eq. (1) without delayed feedback were first studied by Stratonovich [3] by decoupling the correlation and approximating the higher-order correlation functions $C_{n}\left(t^{\prime}\right)=\left\langle x^{n-1}\left(t+t^{\prime}\right) x(t)\right\rangle-\left\langle x^{n-1}\right\rangle\langle x\rangle$ in terms of the usual correlation function $C_{2}\left(t^{\prime}\right)$. The correlation function and correlation time were further investigated 
numerically in [12-14]. The analytical limit of the correlation time at small and large control parameter $a$ are known [15-17]. Furthermore, an analytical expression for the correlation function and correlation time is provided in [17] by using a continued matrix method. This is the method that we have employed in our study and we refer to it as the Jung-Risken theory. The latter has been generalized in [18] for stochastic differential equations with colored noise. The derivation is summarized next in an attempt to use this theory by assuming that the time delay is small in Eq. (1).

\section{CORRELATION TIME}

The correlation time of a stochastic process in its stationary state can be defined as the area under the correlation function normalized by the variance [19],

$$
T=\frac{1}{C(0)} \int_{0}^{\infty} C\left(t^{\prime}\right) d t^{\prime},
$$

where $C\left(t^{\prime}\right)$ is the correlation function,

$$
C\left(t^{\prime}\right)=\left\langle\Delta x\left(t+t^{\prime}\right) \Delta x(t)\right\rangle,
$$

and where $\Delta x=x-\langle x\rangle$. The Jung-Risken theory demonstrates that, given the Markovian process,

$$
\dot{x}(t)=h(x)+g(x) \xi(t),
$$

the correlation time of $x$ can equivalently be written as [17]

$$
T=\frac{1}{C(0)} \int_{0}^{\infty} \frac{f^{2}\left(x^{\prime}\right)}{g^{2}\left(x^{\prime}\right) p_{s}\left(x^{\prime}\right)} d x^{\prime},
$$

where

$$
f(x)=-\int_{0}^{x} \Delta x^{\prime} p_{s}\left(x^{\prime}\right) d x^{\prime},
$$

$g(x)$ is the coefficient of the noise of Eq. (6), and $p_{s}(x)$ is its corresponding stationary probability distribution function.

To apply the Jung-Risken theory to Eq. (1), we need to find the stationary probability distribution function from the Fokker-Planck equation associated with Eq. (1) [2],

$$
\begin{aligned}
\frac{\partial}{\partial t} p(x, t)= & -\frac{\partial}{\partial x}\left\{\left[(a+D) x+b\left\langle x_{\tau} \mid x\right\rangle-x^{3}\right] p(x, t)\right\} \\
& +D \frac{\partial^{2}}{\partial x^{2}}\left[x^{2} p(x, t)\right] .
\end{aligned}
$$

Equation (9) includes a non-Markovian term, the so-called conditional drift,

$$
\left\langle x_{\tau} \mid x\right\rangle=\int_{0}^{\infty} x_{\tau} p\left(x_{\tau} \mid x\right) d x_{\tau} .
$$

To our knowledge, no analytical methods are known to solve this integral. An approximate expression has been given in [6] in the limit of small delay. We directly integrate Eq. (1) on the time interval $t^{\prime} \in[t-\tau, t]$ by using the Stratonovich interpretation of stochastic calculus. The same expression has been obtained from a Taylor expansion of the conditional probability distribution function $p\left(x_{\tau}, t-\tau \mid x, t\right)$ in [2], based on methods introduced in $[20,21]$ under the Ito interpretation of stochastic differential equation. It can also be obtained from a Taylor expansion of the stochastic process defined by $\dot{x}(t, t-\tau)[22,23]$. All methods assume that the time delay is small and lead to, under stationary conditions,

$$
\left\langle x_{\tau} \mid x\right\rangle=[1+\tau(a+b+D)] x-\tau x^{3} .
$$

Equation (11) together with Eq. (9) gives a closed equation for $p(x, t)$,

$$
\begin{aligned}
\frac{\partial}{\partial t} p(x, t)= & -\frac{\partial}{\partial x}\left\{\left[(\sigma+D) x-\gamma x^{3}\right] p(x, t)\right\} \\
& +D \frac{\partial^{2}}{\partial x^{2}}\left[x^{2} p(x, t)\right],
\end{aligned}
$$

where $\sigma=a+b[1+\tau(a+b+D)]$ and $\gamma=(1+b \tau)$. The approximate Fokker-Planck equation [Eq. (12)] is thus Markovian and known results of the Jung-Risken theory can be applied. In particular, the stationary probability distribution function of $x \in[0, \infty]$ satisfies $\dot{p}_{s}(x)=0$ and is found to be

$$
p_{s}(x)=2(\beta \gamma)^{\beta \sigma} \Gamma^{-1}(\beta \sigma)|x|^{2 \beta \sigma-1} e^{-\beta \gamma x^{2}},
$$

where $\beta=(2 D)^{-1}$, and where $\Gamma$ is the gamma function. In those terms, the stochastic threshold is located at $\sigma_{c}=0$. Furthermore, the $n$th moment of the state variable is

$$
\left\langle x^{n}\right\rangle=(\beta \gamma)^{-n / 2} \Gamma^{-1}(\beta \sigma) \Gamma(\beta \sigma+n / 2) .
$$

Substitute Eq. (13) into Eq. (8) so that

$$
f(x)=\langle x\rangle\left[P\left(\beta \sigma, \beta \gamma x^{2}\right)-P\left(\beta \sigma+1 / 2, \beta \gamma x^{2}\right)\right],
$$

where $P(\eta, \omega)$ is the incomplete gamma function defined by

$$
P(\eta, \omega)=\Gamma^{-1}(\eta) \int_{0}^{\omega} q^{\eta-1} e^{-q} d q .
$$

Using the $n=1$ and $n=2$ moments of Eq. (14), together with $g(x)=D^{1 / 2} x$ and Eq. (15), the correlation time corresponding to the random process described by Eq. (12) is

$$
\begin{aligned}
T= & \frac{\beta(\beta \gamma)^{-\beta \sigma} \Gamma(\beta \sigma+1) \Gamma^{2}(\beta \sigma+1 / 2)}{\Gamma^{2}(\beta \sigma+1)-(\beta \sigma) \Gamma^{2}(\beta \sigma+1 / 2)} \\
& \times \int_{0}^{\infty} \frac{\left[P\left(\beta \sigma, \beta \gamma x^{2}\right)-P\left(\beta \sigma+1 / 2, \beta \gamma x^{2}\right)\right]^{2}}{|x|^{2 \beta \sigma+1} e^{-\beta \gamma x^{2}}} d x .
\end{aligned}
$$

Equation (17) has to be evaluated numerically. Because of the singularity at $x=0$, we perform the change of variable $x=\exp (z)$ prior to integration. The calculation has been verified by using several lower bound $z_{\min }$ and upper bound $z_{\max }$; no significant change was observed. The incomplete gamma function is calculated by using an ordinary continued fraction representation [24]. The limits of Eq. (17) are known [15-17]. At large $\sigma$ or small $D$, the correlation time can be expanded in a power series,

$$
T(\sigma, D)=\frac{1}{2 \sigma}+\frac{7 D}{16 \sigma^{2}}+\frac{9 D^{2}}{64 \sigma^{3}}+O\left[\frac{D^{3}}{\sigma^{4}}\right],
$$

whereas for small $\sigma$ or large $D$,

$$
T(\sigma, D)=\frac{\pi}{2 \sigma}-\frac{A}{D}+O\left[\frac{\sigma}{D^{2}}\right],
$$




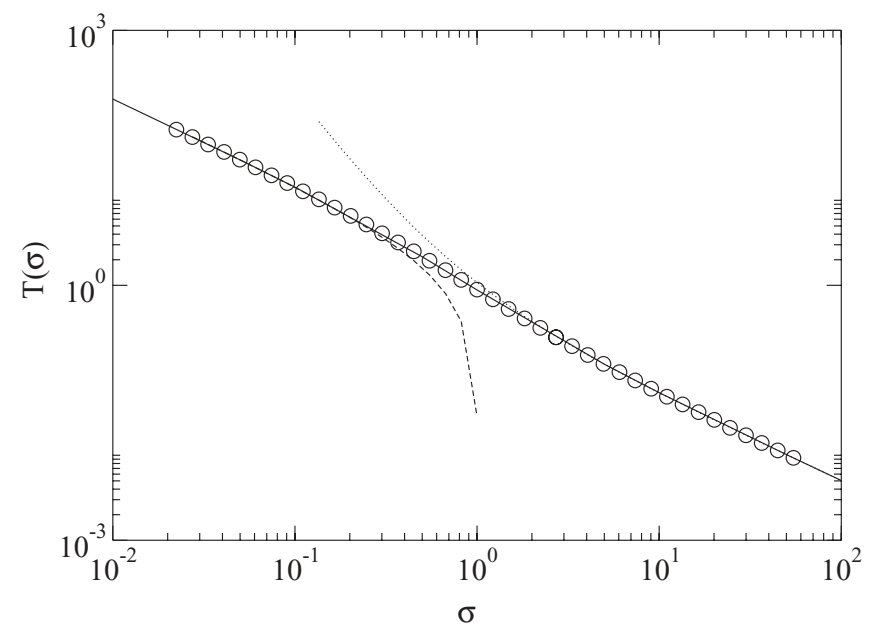

FIG. 1. Correlation time $T(\sigma)$ as a function of the parameter $\sigma=a+b[1+\tau(a+b+D)]$ as calculated from Eq. (17) at fixed $b=0$ and $D=1$ (symbols). The dotted curve is the large- $\sigma$ limit [Eq. (18)] and the dashed curve is the limit for small $\sigma$ [Eq. (19)]. The solid curve is the Padé approximation [Eq. (20)].

where $A=1.5421 \ldots$... A Padé approximation to Eq. (17) has also been proposed in [17]. The expression is

$$
T(\sigma, D)=\frac{1}{2 \sigma}+\frac{0.6037 D \sigma+1.0708 D^{2}}{1.38 \sigma^{3}+2.004 D \sigma^{2}+D^{2} \sigma} .
$$

The correlation time $T$ obtained from the numerical integration of Eq. (17) is shown in Fig. 1, together with its asymptotic limits of small and large $\sigma$. We also show the Padé interpolant. Equation (20) is expected to be valid in the limit of small time delay. We will compare this result with the correlation time computed from a direct numerical solution of Eq. (1) in the next section.

\section{NUMERICAL EVALUATION OF THE CORRELATION TIME}

The correlation function and correlation time are determined numerically with finite feedback, and compared to the approximation that leads to a Jung-Risken determination. Equation (1) is numerically integrated by using a secondorder integration algorithm that includes delayed feedback as derived in [2]. The initial condition is a constant function in $[-\tau, 0]$ for each trajectory, with the constant being drawn from a Gaussian distribution of zero mean and variance 1 . The time step used in the numerical integration is $\Delta t=0.01$. The correlation function of the dynamical variable $x$,

$$
C\left(t^{\prime}\right)=\left\langle x\left(t+t^{\prime}\right) x(t)\right\rangle-\langle x\rangle^{2},
$$

is computed in the time interval $t \in\left[t_{\min }, t_{\max }\right]$, with $t_{\min }=300$ and $t_{\max }=1400$. Trajectories have reached a stationary state in this time interval. Note that the lower bound $t_{\min }$ is larger than the largest correlation time that we have calculated. For a given trajectory, the correlation function is averaged over $N=10^{4}$ values of $x\left(t_{i}\right)$, with $t_{i}=\left\{t_{\min }, \ldots, t_{\min }+N \Delta t\right\}$, at fixed time lag $t^{\prime}$. The correlation function is constructed for a time lag $t^{\prime} \in[0, L]$, where $L$ is the maximum time displacement. The ensemble average is further constructed by considering $10^{6}$ independent trajectories. The first moment of the state variable $x$ in Eq. (21) is calculated in the time interval $t \in\left[t_{\min }, t_{\min }+\right.$ $L+N \Delta t]$. The correlation time is the area under the curve of the normalized correlation function. It is calculated according to Eq. (4) by using an integration time step of $\Delta t^{\prime}=0.01$.

Close to threshold, the correlation time is expected to diverge. However, due to the finite integration time window involved in the numerical integration, a true divergence cannot occur. In order to characterize the divergence given the finite size of the integration domain, we repeat the calculation of the correlation function for several values of the maximum time lag $L=\{20,50,100,200,500\}$. The procedure is repeated for different values of the control parameter $a$ close to threshold. In what follows, we present the results with delayed feedback and compare them to the known case of no delay $(b=0)$. The calculations are also repeated at different values of the time delay $\tau$. In this paper, we limit our analysis to the pitchfork branch of Eq. (1) $(b \tau>-1)$.

\section{A. No delay $(b=0)$}

The correlation time without delay $(b=0)$ as calculated from the normalized correlation function is shown in Fig. 2 . Away from the bifurcation threshold $\left(\sigma \gg \sigma_{c}=0\right)$, the normalized correlation function decays rapidly to zero. For large values of the control parameter, trajectories quickly saturate and fluctuate around their equilibrium value. The correlation time is small for this range of parameters and agrees well with the analytical prediction [Eq. (17)]. The decay of the normalized correlation function slows down as the bifurcation threshold is approached $\left(\sigma \sim \sigma_{c}\right)$. In this range, one would have to integrate the normalized correlation function over a very long time lag window to approach the analytical prediction. We use instead scaling theory to overcome this limitation and to characterize the divergence of the correlation time.

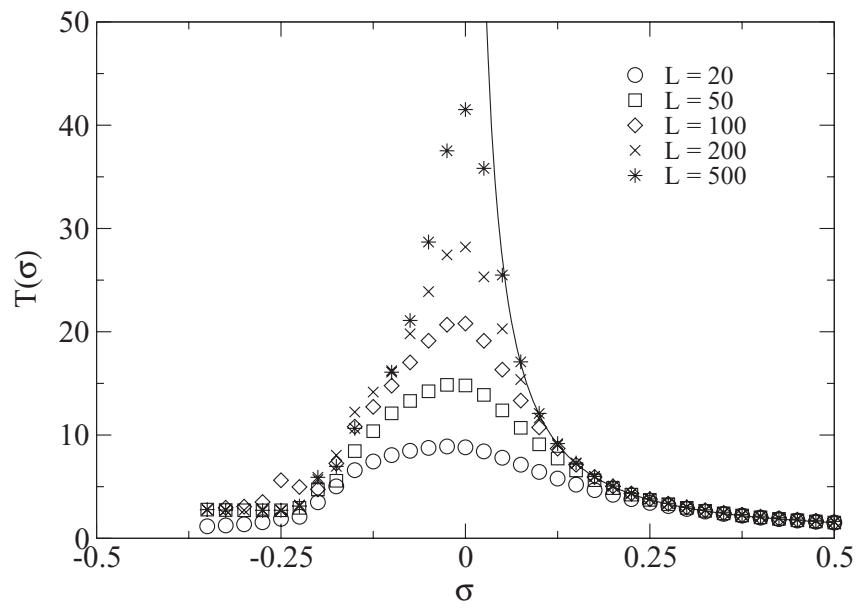

FIG. 2. Correlation time $T(\sigma)$ as a function of the parameter $\sigma$ without delayed feedback $(b=0)$ at fixed $D=0.3$. The symbols are the numerically determined correlation times for several maximum time lags $L$ while the solid line is the Padé approximation [Eq. (20)]. The stochastic bifurcation is located at $\sigma_{c}=0$. 


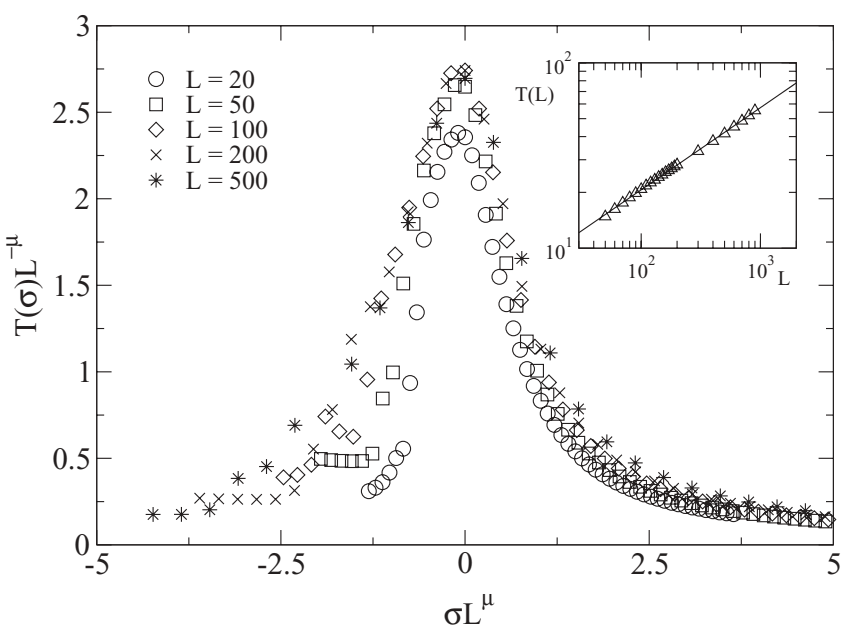

FIG. 3. Correlation time scaled by a power law of the maximum time lag $T(\sigma) L^{-\mu}$ as a function of the parameter $\sigma$ also scaled by a power law of the maximum time lag $\sigma L^{\mu}$ without delayed feedback $(b=0)$ at fixed $D=0.3$. The inset shows the correlation time $T(L)$ evaluated at threshold $\left(\sigma_{c}=0\right)$ as a function of the maximum time lag $L$ for the same set of parameters. The solid curve is $T(L)=$ $2.69 L^{0.443}$. We use this linear regression to scale the correlation time, which is evaluated at $\mu=0.44$, the best estimate for the exponent in the limit $L \rightarrow \infty$.

The correlation time is expected to diverge at threshold $\left(\sigma \sim \sigma_{c}\right)$ for $L \rightarrow \infty$. We therefore write for finite $L$,

$$
T(\sigma, L)=L^{\mu} \tilde{T}\left(\sigma L^{\nu}\right)
$$

For finite $\sigma$ and taking the limit $L \rightarrow \infty$, we have that $T \sim \sigma^{-\mu / v}$ if the scaling function is regular. Close to threshold, Eq. (19) predicts that the correlation time diverges with exponent $\mu / \nu=1$. We show in Fig. 3 the scaled correlation time assuming only one unknown exponent for $L \in[50,900]$. Furthermore, the value of the exponent $\mu$ is determined from the dependence of the correlation time on the maximum time lag evaluated at threshold $\left(\sigma_{c}=0\right)$. The extrapolation is shown in the inset of Fig. 3. The correlation time appears to follow a power law with the maximum time lag. Our best estimate for the exponent is $\mu=v=0.44$. It is this value of the exponent that has been used to scale the correlation time according to Eq. (22), shown in Fig. 3. With this exponent, the curves of the correlation time calculated at different maximum time lag collapse to each other close to the bifurcation threshold. We conclude that the correlation time follows a power law divergence close to threshold, with exponent $T \sim \sigma^{-1}$. Therefore, the stochastic bifurcation in the absence of delay is sharp.

\section{B. Delayed feedback}

A similar analysis to that presented above is repeated for the case with delay $(b \neq 0)$. Delayed feedback induces the presence of additional peaks in the normalized correlation function of the dynamical variable $x$ as shown in Fig. 4. These peaks are a direct consequence of the correlation between the state of $x$ at times $t$ and $t-\tau$ introduced by the delay. The correlation time with delayed feedback is calculated from a

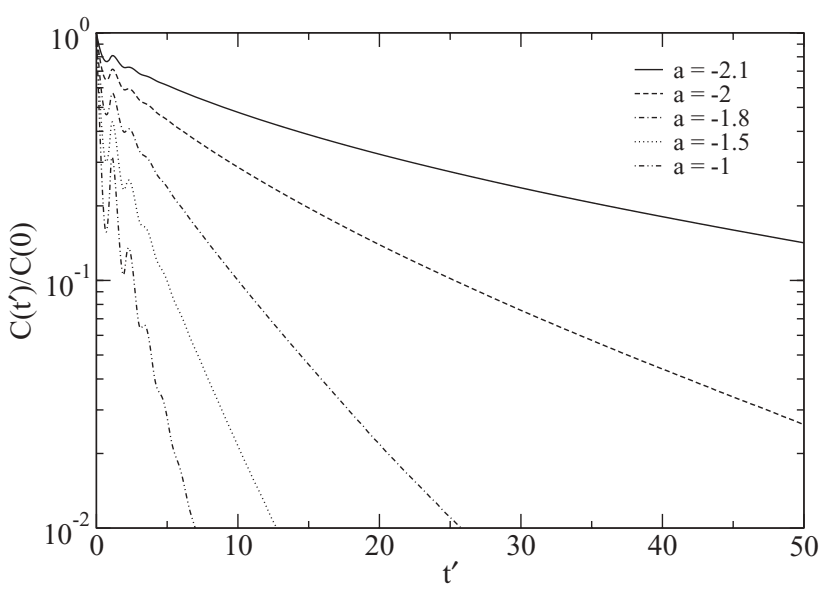

FIG. 4. Correlation function $C\left(t^{\prime}\right)$ normalized by the variance $C(0)$ of the dynamical variable $x$ in its stationary state as a function of the time lag $t^{\prime}$ at fixed $b=2, D=0.3$, and $\tau=1$, as the control parameter $a$ is varied. The bifurcation threshold is located at $a_{c}=$ -2.2 for this set of parameters. Time delay induces peaks in the correlation function because the state of the dynamical variable $x$ at time $t$ is correlated with its value at time $t-\tau$.

direct numerical integration of the governing equations and is shown in Fig. 5 for different values of $L$ together with the Padé approximation given in Eq. (20). The analytical determination of the threshold agrees well with the location of the maximum of $T$ in the figure. As was the case for no delay $(b=0)$, the maximum of the numerically determined correlation time increases with $L$. The scaling form [Eq. (22)] is tested again with the same condition $\mu=v$ that follows from our analytic results for small $\tau$. We show our scaling results with $\sigma_{c}=0$ or $a_{c}=-2.2$ in Fig. 6 . We further estimate $\mu \approx 0.53$ by fitting the correlation time as a function of $L \in[50,900]$ (inset in the figure). This value of the exponent is quite close to the case of no delay. The correlation time curves calculated for different values of $L$ collapse close to the bifurcation threshold as shown

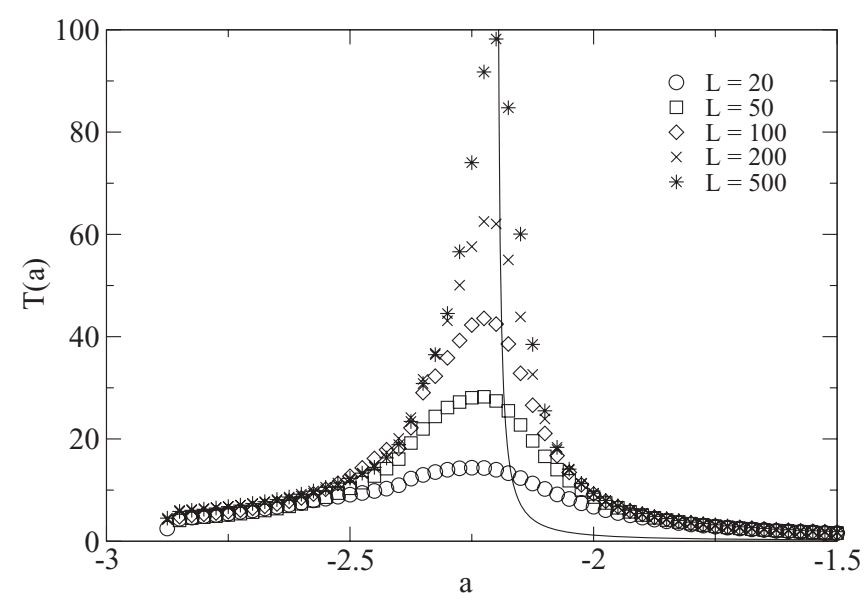

FIG. 5. Correlation time $T(a)$ as a function of the control parameter $a$ with $b=2, \tau=1$, and $D=0.3$. Results for several maximum time lags $L$ are shown. The solid line is the Padé approximation [Eq. (20)]. For those parameters, the bifurcation threshold is located at $a_{c}=-2.2$, in agreement with the maximum of the correlation time and the divergence of the Padé approximation. 


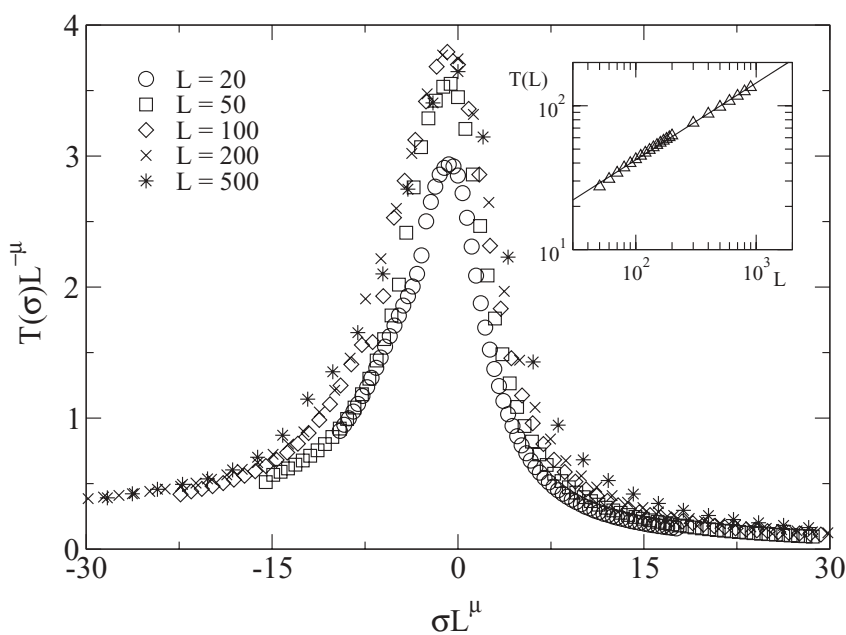

FIG. 6. Correlation time scaled by a power law of the maximum time lag $T(\sigma) L^{-\mu}$ as a function of the parameter $\sigma$ also scaled by a power law of the maximum time $\operatorname{lag} \sigma L^{\mu}$ with $b=2, \tau=1, D=0.3$, and with exponent $\mu=0.53$. The stochastic bifurcation threshold is located at $\sigma_{c}=0$. The inset shows the correlation time $T(L)$ evaluated at threshold as a function of the maximum time lag $L$ for the same set of parameters. The solid curve is $T(L)=3.60 L^{0.534}$.

in Fig. 6. We therefore conclude that the correlation time diverges as $T \sim \sigma^{-1}$. Interestingly, time delay does not change the nature of the bifurcation or the value of the exponents. The bifurcation remains sharp as was suggested in [2].

We finally note that the value of the correlation time predicted from Eq. (17) does not agree with our numerical determination, only the location of the threshold. According to our analytic prediction, the correlation time depends on delay $\tau$ through $\sigma$. This is not the case for our numerical results as $T$ has a separate dependence on $\tau$ as shown in Fig. 7. Delayed feedback induces correlations between the state of the dynamical variable $x$ at times $t$ and $t-\tau$ and those correlations affect the value of the normalized correlation function. Those additional correlations are not included in the

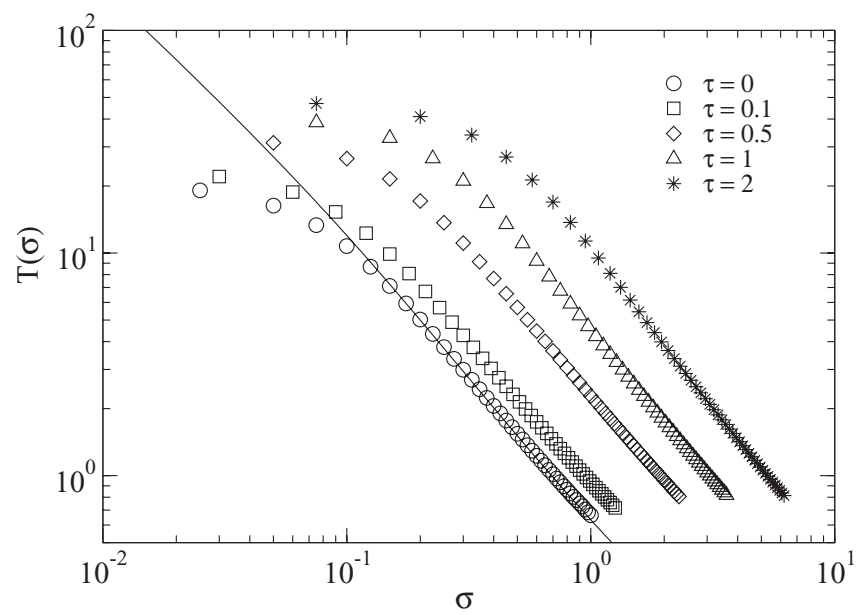

FIG. 7. Correlation time $T(\sigma)$ as a function of $\sigma=a+b[1+$ $\tau(a+b+D)]$ with $b=2$ and $D=0.3$ for different values of the time delay $\tau$. The solid curve is the Padé approximation [Eq. (20)]. The magnitude of the numerically determined correlation time at $\tau \neq 0$ disagrees with the Padé approximation at the same parameters due to the non-Markovian nature of the delay stochastic differential equation under study.

Jung-Risken theory applied to small delay because it assumes that the transition probabilities are Markovian.

\section{ACKNOWLEDGMENTS}

This research has been supported by NSERC Canada. M.G. acknowledges funding by Le Fonds Québécois de la Recherche sur la Nature et les Technologies (FQRNT). J.M.B. acknowledges the Graduate Students' Exchange Program (GSEP) offered by the Canadian Bureau for International Education (CBIE) and the Brazilian agencies CNPq (Grant No. 134461/2007-0) and FAPESP (Grant No. 2009/11567-6) for financial support. We thank two Compute Canada sites, CLUMEQ and SciNet, for access to supercomputing resources. Compute Canada is supported by the Canada Foundation for Innovation.
[1] D. Bratsun, D. Volfson, L. Tsimring, and J. Hasty, Proc. Natl. Acad. Sci. USA 102, 14593 (2005).

[2] M. Gaudreault, F. Lépine, and J. Viñals, Phys. Rev. E 80, 061920 (2009).

[3] R. Stratonovich, Topics in the Theory of Random Noise (Gordon and Breach, London, 1967), Vol. 2.

[4] M. C. Mackey and I. G. Nechaeva, Phys. Rev. E 52, 3366 (1995).

[5] J. Smythe, F. Moss, P. V. E. McClintock, and D. Clarkson, Phys. Lett. A 97, 95 (1983).

[6] M. Gaudreault, F. Drolet, and J. Viñals, Phys. Rev. E 82, 051124 (2010).

[7] R. Graham and A. Schenzle, Phys. Rev. A 25, 1731 (1982).

[8] F. Drolet and J. Viñals, Phys. Rev. E 57, 5036 (1998).

[9] M. San Miguel and R. Toral, in Instabilities and Nonequilibrium Structures VI, edited by E. Tirapegui, J. Martinez, and R. Tiemann (Kluwer Academic, Dordrecht, 2000).
[10] U. Küchler and B. Mensch, Stoch. Stoch. Rep. 40, 23 (1992).

[11] T. Ohira and T. Yamane, Phys. Rev. E 61, 1247 (2000).

[12] J. M. Sancho, M. San Miguel, S. L. Katz, and J. D. Gunton, Phys. Rev. A 26, 1589 (1982).

[13] J. M. Sancho, R. Mannella, P. V. E. McClintock, and F. Moss, Phys. Rev. A 32, 3639 (1985).

[14] R. Mannella, S. Faetti, P. Grigolini, and P. McClintock, J. Phys. A: Math. Gen. 21, 1239 (1988).

[15] S. Faetti, C. Festa, L. Fronzoni, P. Grigolini, F. Marchesoni, and V. Palleschi, Phys. Lett. A 99, 25 (1983).

[16] A. Hernandez-Machado, M. San Miguel, and J. M. Sancho, Phys. Rev. A 29, 3388 (1984).

[17] P. Jung and H. Risken, Z. Phys. B 59, 469 (1985).

[18] J. Casademunt, R. Mannella, P. V. E. McClintock, F. E. Moss, and J. M. Sancho, Phys. Rev. A 35, 5183 (1987). 
[19] W. Hornsthemke and R. Lefever, Noise Induced Transitions: Theory and Applications in Physics, Chemistry, and Biology (Springer, Berlin, 1984).

[20] T. D. Frank, Phys. Rev. E 72, 011112 (2005).

[21] H. Risken, The Fokker-Planck Equation: Methods of Solution and Applications (Springer, Berlin, 1989).
[22] S. Guillouzic, I. L'Heureux, and A. Longtin, Phys. Rev. E 59, 3970 (1999).

[23] S. Guillouzic, I. L'Heureux, and A. Longtin, Phys. Rev. E 61, 4906 (2000).

[24] Handbook of Mathematical Functions, edited by M. Abramowitz and I. Stegun (Dover, New York, 1964). 